\title{
Visibility of dichalcogenide nanolayers
}

\author{
M M Benameur ${ }^{1}$, B Radisavljevic ${ }^{1}$, J S Héron ${ }^{1}$, S Sahoo ${ }^{1}$, H Berger ${ }^{2}$ \\ and $\mathrm{A} \mathrm{Kis}^{1}$ \\ ${ }^{1}$ Electrical Engineering Institute, School of Engineering, EPFL, CH-1015 Lausanne, \\ Switzerland \\ 2 Institute of Condensed Matter Physics, School of Basic Science, EPFL, CH-1015 Lausanne, \\ Switzerland
}

Received 19 October 2010, in final form 9 January 2011

Published 14 February 2011

Online at stacks.iop.org/Nano/22/125706

\begin{abstract}
Dichalcogenides with the common formula $\mathrm{MX}_{2}$ are layered materials with electrical properties that range from semiconducting to superconducting. Here, we describe optimal imaging conditions for the optical detection of ultrathin, two-dimensional dichalcogenide nanocrystals containing single, double and triple layers of $\mathrm{MoS}_{2}, \mathrm{WSe}_{2}$ and $\mathrm{NbSe}_{2}$. A simple optical model is used to calculate the contrast for nanolayers deposited on wafers with varying thicknesses of $\mathrm{SiO}_{2}$. The model is extended for imaging using the green channel of a video camera. Using AFM and optical imaging we confirm that single layers of $\mathrm{MoS}_{2}$ and $\mathrm{WSe}_{2}$ can be detected on 90 and $270 \mathrm{~nm} \mathrm{SiO} 2$ using optical means. By measuring contrast under broadband green illumination we are also able to distinguish between nanostructures containing single, double and triple layers of $\mathrm{MoS}_{2}$ and $\mathrm{WSe}_{2}$. We observe and discuss discrepancies in the case of $\mathrm{NbSe}_{2}$.
\end{abstract}

(Some figures in this article are in colour only in the electronic version)

The family of transition metal dichalcogenides with the common formula $\mathrm{MX}_{2}$, where $\mathrm{M}$ stands for transition metal $(\mathrm{M}=\mathrm{Mo}, \mathrm{W}, \mathrm{Nb}, \mathrm{Ta}, \mathrm{Ti})$ and $\mathrm{X}$ for $\mathrm{Se}, \mathrm{S}$ or Te displays a rich variety of physical properties. Depending on the metal and the chalcogen involved, their electrical properties span the range from semiconducting to superconducting. Bulk dichalcogenide crystals are composed of vertically stacked layers bound together by weak van der Waals interaction. Just as in the case of graphene [1], single dichalcogenide layers can be extracted from bulk crystals [2,3] and deposited on substrates for further studies. Single $\mathrm{MX}_{2}$ layers present a wide range of systems for studying mesoscopic transport in 2D and could find practical applications complementary to those of graphene. Bulk $\mathrm{WSe}_{2}$ has, for example, been used in the past for fabrication of photovoltaic cells [4], whereas $\mathrm{MoS}_{2}$ nanotubes [5] and nanowires [6] show confinement effects in their electronic and optical properties. Semiconducting dichalcogenides could also be interesting for fabrication of nanoscale field effect transistors [3, 7-9] while superconducting $\mathrm{NbSe}_{2}$ could be a model for studying superconductivity in low-dimensional systems at mesoscopic scales [10, 11].

Locating and identifying single nanolayers of materials such as graphite [1] or semiconducting transition metal dichalcogenides [3] such as $\mathrm{MoS}_{2}$ or $\mathrm{WSe}_{2}$ is the first, enabling step in the study and practical applications of these materials. Atomic force microscopy (AFM) can be used to accurately determine both the vertical and lateral dimensions of nanolayers deposited on insulating substrates such as $\mathrm{SiO}_{2}$. AFM imaging is, however, time-consuming and the relatively slow throughput of the technique is a serious drawback. Scanning electron microscopy (SEM) or transmission electron microscopy (TEM) could also be used here, but contamination [12] due to electron-beam-induced deposition or knock-on damage in TEM due to electron-beam radiation-induced displacement of atoms could be a serious problem here.

Optical imaging offers the possibility of simple, rapid and non-destructive characterization of large-area samples. In the case of graphene deposited on $\mathrm{SiO}_{2}$, it has been found that even the presence of a single layer can produce a detectable contrast with respect to the interference color of the underlying oxide layer $[13,14]$. The simplicity and accessibility of this detection scheme was one of the most important factors that allowed the rapid spread in graphene-related research.

It is not clear what would be the optimal oxide thickness for the optical detection of dichalcogenide nanolayers. It could even be possible that such nanolayers deposited on $300 \mathrm{~nm} \mathrm{SiO} 2$, commonly used for graphene-related studies, might be invisible because of a particularly unfortunate set of interference conditions.

We have therefore decided to calculate the contrast for several different types of nanolayers deposited on $\mathrm{SiO}_{2}$ 


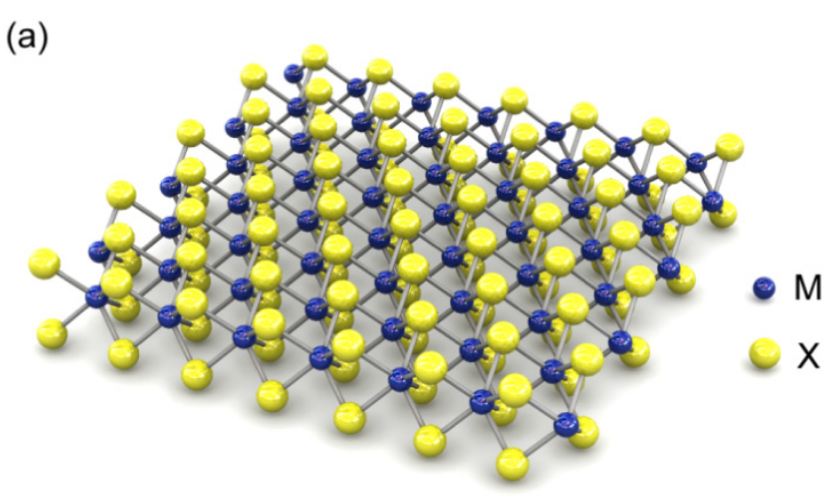

(b)

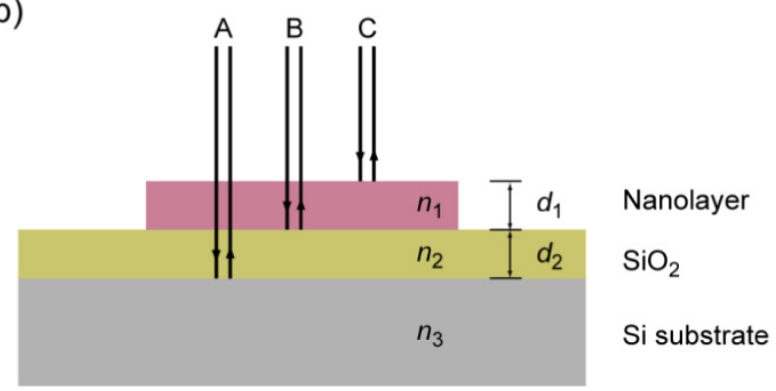

Figure 1. (a) Three-dimensional representation of a dichalcogenide monolayer with a generic formula $\mathrm{MX}_{2}$. (b) Schematic depiction of optical reflection and transmission for nanolayer with thickness $d_{1}$ and complex index of refraction $n_{1}$ deposited on an $\mathrm{SiO}_{2}$ layer characterized by thickness $d_{2}$ and index of refraction $n_{1}$ that is grown on top of a degenerately doped Si substrate. Nanolayers deposited on $\mathrm{SiO}_{2}$ are visible due to interference between light rays $\mathrm{A}, \mathrm{B}$ and $\mathrm{C}$ reflected at various interfaces in the stack.

in order to determine the optimal imaging conditions for their optical detection. In this work we focus on three representative dichalcogenide materials that might be most interesting for future studies: semiconducting $\mathrm{MoS}_{2}$ and $\mathrm{WSe}_{2}$ that could be useful for fabrication of nanoscale field effect transistors [3, 7-9] and superconducting $\mathrm{NbSe}_{2}$ which could be a new model for studying superconductivity in lowdimensional systems $[10,11]$.

In analogy with graphene [13-16], the contrast between dichalcogenide nanolayers such as the one depicted on figure 1, and the underlying $\mathrm{SiO}_{2}$ substrate is due to a phase shift of the interference color and material opacity. In order to calculate this contrast, we consider the stacking of two thin films (2D dichalcogenide material and $\mathrm{SiO}_{2}$ ) on top of a third semiinfinite film (degenerately doped n-type $\mathrm{Si}$ ), as depicted on figure 1 . The 2D nanolayer is modeled as a thin homogeneous film of thickness $d_{1}$ with complex refractive index $n_{1}$, where $\operatorname{Re}\left(n_{1}\right)$ is the optical refractive index and $-\operatorname{Im}\left(n_{1}\right)$ is the absorption coefficient. Previously published values for the refractive indices and absorption coefficients of bulk $\mathrm{MoS}_{2}$, $\mathrm{WSe}_{2}$ and $\mathrm{NbSe}_{2}$ are available in the literature [17-19]. The $\mathrm{SiO}_{2}$ layer of thickness $d_{2}$ is optically characterized by a wavelength-dependent refractive index $n_{2}(\lambda)$ with only a real part [20], ranging from 1.47 at $400 \mathrm{~nm}$ to 1.455 at $700 \mathrm{~nm}$. As the thickness of the degenerately doped Si layer $(525 \mu \mathrm{m})$ is several orders of magnitude larger than the corresponding skin depth, it can be considered as a semi-infinite film. For normal light incidence, the intensity of reflected light from the stacking of two thin films on top of a semi-infinite layer is given by $[13,21]$

$R\left(n_{1}\right)$

$=\left|\frac{r_{1} \mathrm{e}^{\mathrm{i}\left(\phi_{1}+\phi_{2}\right)}+r_{2} \mathrm{e}^{-\mathrm{i}\left(\phi_{1}-\phi_{2}\right)}+r_{3} \mathrm{e}^{-\mathrm{i}\left(\phi_{1}+\phi_{2}\right)}+r_{1} r_{2} r_{3} \mathrm{e}^{\mathrm{i}\left(\phi_{1}-\phi_{2}\right)}}{\mathrm{e}^{\mathrm{i}\left(\phi_{1}+\phi_{2}\right)}+r_{1} r_{2} \mathrm{e}^{-\mathrm{i}\left(\phi_{1}-\phi_{2}\right)}+r_{1} r_{3} \mathrm{e}^{-\mathrm{i}\left(\phi_{1}+\phi_{2}\right)}+r_{2} r_{3} \mathrm{e}^{\mathrm{i}\left(\phi_{1}-\phi_{2}\right)}}\right|^{2}$

where

$$
r_{1}=\frac{n_{0}-n_{1}}{n_{0}+n_{1}}, \quad r_{2}=\frac{n_{1}-n_{2}}{n_{1}+n_{2}}, \quad r_{3}=\frac{n_{2}-n_{3}}{n_{2}+n_{3}}
$$

are the relative indices of refraction and $\phi_{i}=\frac{2 \pi d_{i} n_{i}}{\lambda}$ are the phase shifts induced by changes in the optical path.

On the other hand, the reflected light intensity in the absence of a nanolayer can be found by substituting $n_{1}=1$ :

$$
R\left(n_{1}=1\right)=\left|\frac{r_{2}^{\prime} \mathrm{e}^{\mathrm{i}\left(\phi_{2}\right)}+r_{3} \mathrm{e}^{-\mathrm{i}\left(\phi_{2}\right)}}{\mathrm{e}^{\mathrm{i}\left(\phi_{2}\right)}+r_{2}^{\prime} r_{3} \mathrm{e}^{-\mathrm{i}\left(\phi_{2}\right)}}\right|^{2}
$$

where $r_{2}^{\prime}=\frac{n_{0}-n_{2}}{n_{0}+n_{2}}$ is the relative index of refraction at the interface between air and the dielectric thin film.

The contrast is defined as the relative intensity of reflected light in the presence and absence of the $2 \mathrm{D}$ dichalcogenide material and can be written as

$$
\text { Contrast }=\frac{R\left(n_{1}=1\right)-R\left(n_{1}\right)}{R\left(n_{1}=1\right)} .
$$

In order to determine optimal conditions for the optical detection of nanolayers we plot the calculated contrast as a function of incident light wavelength and $\mathrm{SiO}_{2}$ thickness in figure 2. For all three materials and $\mathrm{SiO}_{2}$ thickness lower than $300 \mathrm{~nm}$, the contrast for visible light wavelengths exhibits two characteristic bands with high, positive contrast and one band with negative contrast. The two bands with positive contrast roughly correspond to $\mathrm{SiO}_{2}$ thickness in the 50-100 nm and 200-300 $\mathrm{nm}$ range, implying that dichalcogenide nanolayers should, in principle, be visible on substrates with such oxide thicknesses for at least some spectral ranges of the visible light. In the 130-160 $\mathrm{nm} \mathrm{SiO}_{2}$ thickness range, we expect to see weaker, negative contrast. In the $0-50 \mathrm{~nm}, 100-130 \mathrm{~nm}$ and 160-200 nm regions we expect the contrast to be too low $(<5-$ $10 \%$ ) for the flakes to be visible.

In the next step, we generalize the model for broadband illumination by recognizing that typical color cameras contain red, green and blue color filters. This allows us to calculate contrast values observed with standard color cameras and white light illumination, avoiding the need for narrow-band color filters tuned to a specific color range [13] or expensive instrumentation such as confocal microscopes [14]. We can compute the effective contrast by calculating the average contrast weighed by the camera response function $S(\lambda)$ for a given channel (red, green or blue). The response function is available in technical specifications for a given camera and is primarily determined by the Bayer filter in front of the camera's $\mathrm{CCD}$, implying that our findings are relevant to color cameras from other manufacturers. We limit ourselves to the green 

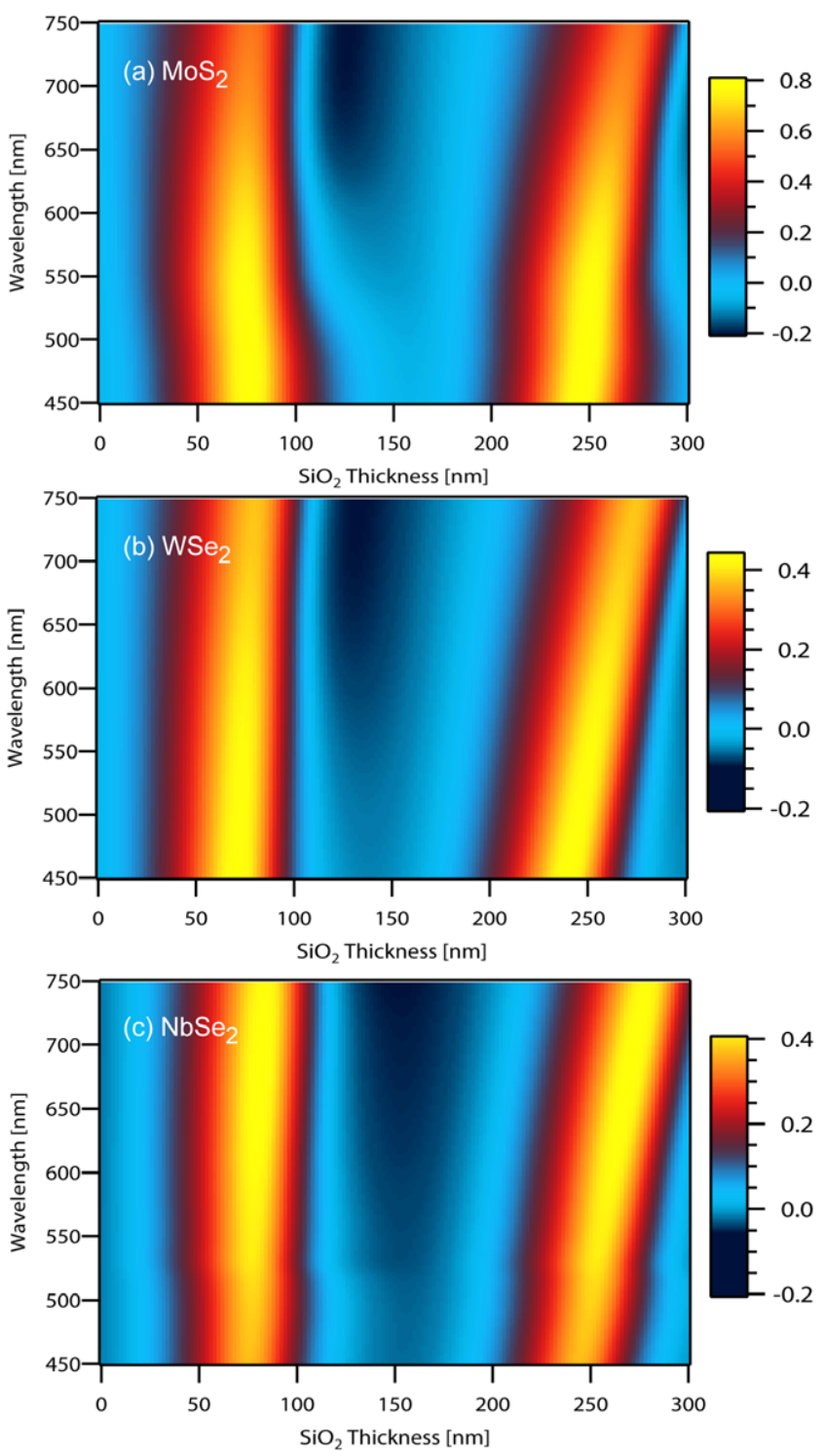

Figure 2. Color plot of calculated contrast as a function of incident light wavelength and $\mathrm{SiO}_{2}$ layer thickness for (a) $\mathrm{MoS}_{2}$, (b) $\mathrm{WSe}_{2}$ and (c) $\mathrm{NbSe}_{2}$. Dichalcogenide nanolayers are expected to be visible on substrates with oxide thickness in the $50-100 \mathrm{~nm}$ and 200-300 $\mathrm{nm}$ range. In the $100-150 \mathrm{~nm} \mathrm{SiO}_{2}$ thickness range, we expect to see weaker, negative contrast for red light illumination.

channel only (495-530 nm), as the typical Bayer filter used in color cameras contains $50 \%$ green and only $25 \%$ of red and blue elements each, implying that the green channel is expected to contain less noise. The contrast in the green channel is then given by

$\operatorname{Contrast}_{\text {green }}\left(d_{\text {substrate }}\right)$

$$
=\frac{\int_{\lambda=495 \mathrm{~nm}}^{\lambda=530 \mathrm{~nm}} S(\lambda) \operatorname{Contrast}\left(\lambda, d_{\text {substrate }}\right) \mathrm{d} \lambda}{\int_{\lambda=495 \mathrm{~nm}}^{\lambda=530 \mathrm{~nm}} S(\lambda) \mathrm{d} \lambda} .
$$

Calculated values are reported in figure 3 . For all the three materials that we studied, we find three characteristic peaks in the $0-300 \mathrm{~nm}$ region. Among these peaks, two of them show positive contrast values while one of them shows negative contrast. In the case of positive contrast the thin nanolayers
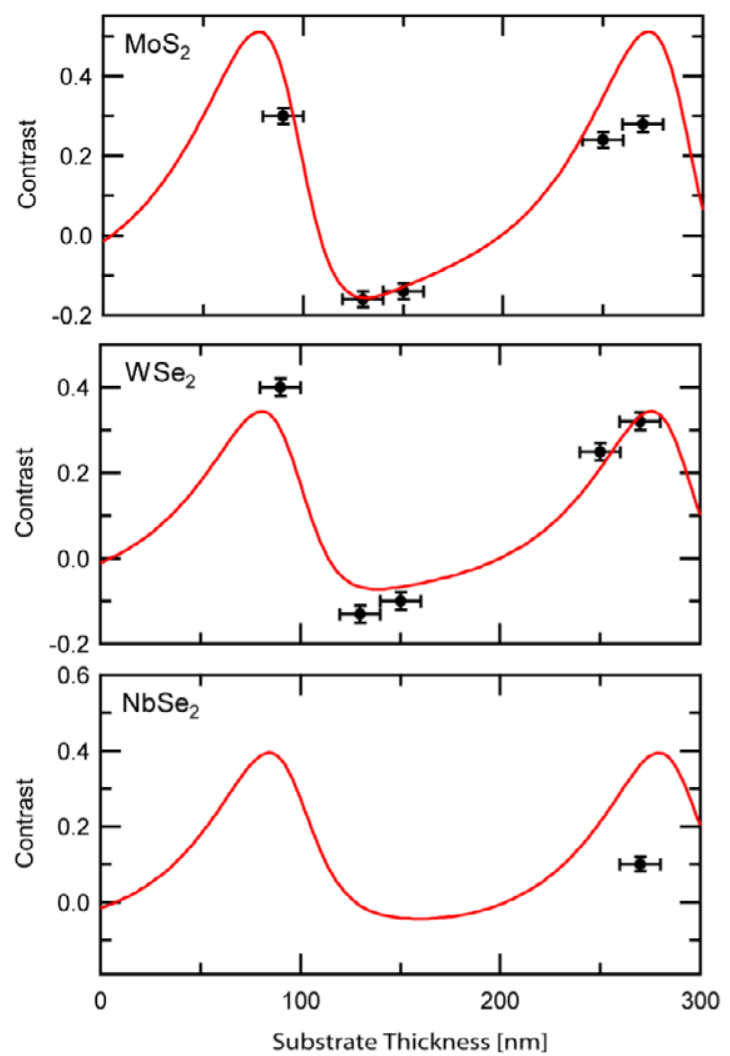

Figure 3. Calculated contrast values for $\mathrm{MoS}_{2}, \mathrm{WSe}_{2}$ and $\mathrm{NbSe}_{2}$ deposited on $\mathrm{SiO}_{2}$ substrates with varying thicknesses. Curves represent contrast for broadband illumination and detection using the green channel (495-530 nm) of a color camera. Black dots are experimental data points.

appear darker than the substrate while in the case of negative contrast they appear brighter.

The two peaks with positive contrast are located at 78 and $272 \mathrm{~nm}$ in the case of $\mathrm{MoS}_{2}$, at 80 and $274 \mathrm{~nm}$ for $\mathrm{WSe}_{2}$ and at 84 and $274 \mathrm{~nm}$ for $\mathrm{NbSe}_{2}$. The peak with negative contrast is located at $132 \mathrm{~nm}$ for $\mathrm{MoS}_{2}$, at $138 \mathrm{~nm}$ for $\mathrm{WSe}_{2}$ and at $160 \mathrm{~nm}$ for $\mathrm{NbSe}_{2}$.

Based on these calculations, we predict that substrates with $\mathrm{SiO}_{2}$ thicknesses of 90, 140 and $270 \mathrm{~nm}$ should result in sufficient contrast $(\mid$ Contrast $\mid \geqslant 10 \%)$ for optical detection of dichalcogenide nanolayers. We note that the 90 and $270 \mathrm{~nm}$ $\mathrm{SiO}_{2}$ thicknesses are sufficiently close to optimal conditions for imaging graphene (90 and $280 \mathrm{~nm}$ ) [13]. Using a standard microscope with a color camera and white light illumination also results in more flexibility, as it allows us to distinguish between scotch-tape residue and nanolayers more easily than by using a monochromatic image alone. Even though scotchtape residue has a similar contrast in the green region as dichalcogenide nanolayers, this is not true for other channels (red and blue), making it easy to distinguish the two by comparing their colors alone. Our set-up is also less expensive and more accessible then confocal microscopes.

We proceed by depositing individual dichalcogenide nanolayers on substrates with 90, 130, 150, 250 and $270 \mathrm{~nm}$ $\mathrm{SiO}_{2}$ thickness using the mechanical exfoliation technique commonly used for graphene deposition [1]. Briefly, we 


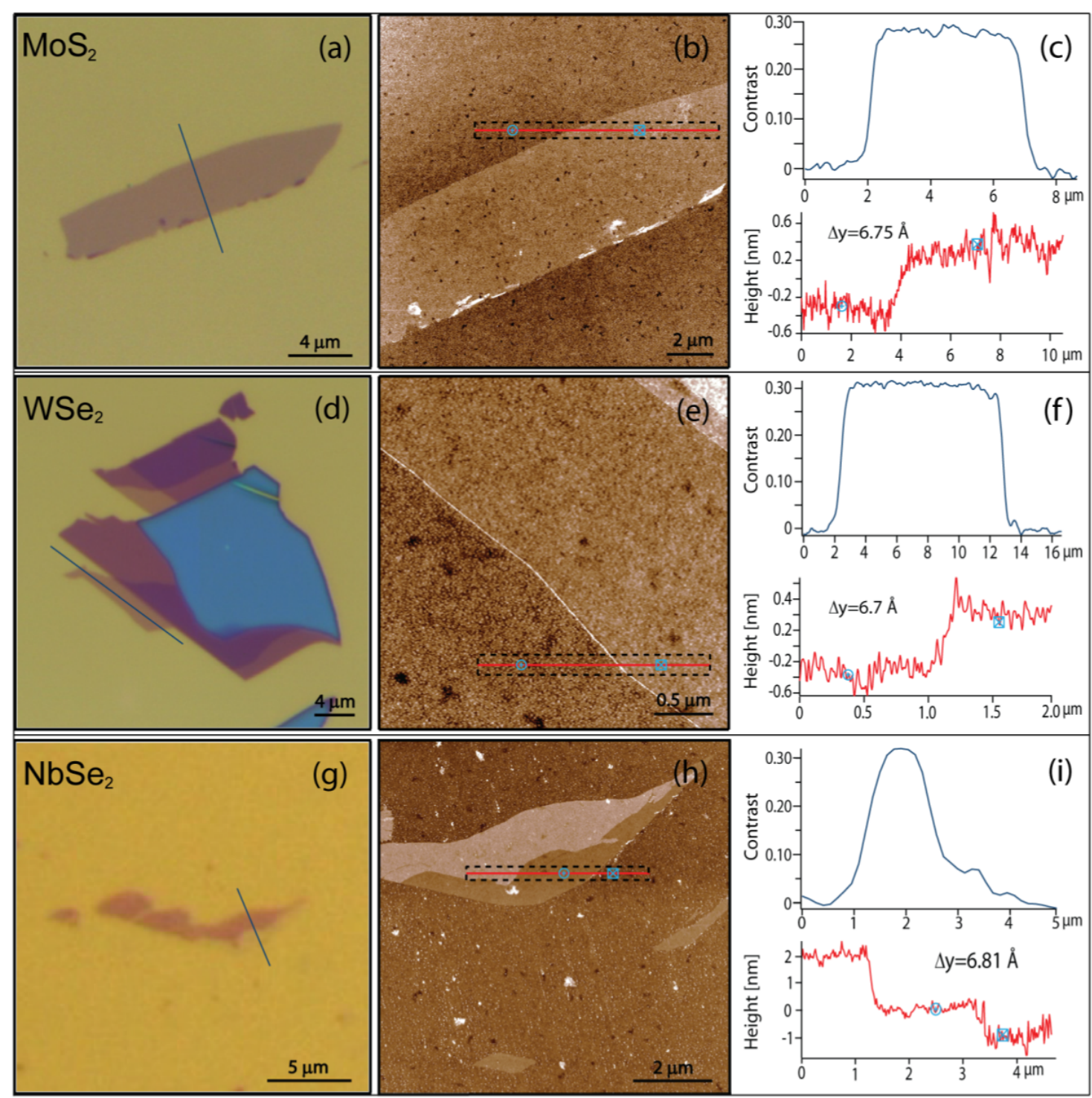

Figure 4. Optical and AC mode AFM images of dichalcogenide nanolayers deposited on $270 \mathrm{~nm} \mathrm{SiO}_{2}$ with corresponding contrast and height profiles of monolayers: (a)-(c) for $\mathrm{MoS}_{2}$, (d)-(f) for $\mathrm{WSe}_{2}$ and (g)-(i) for $\mathrm{NbSe}_{2}$. Contrast and height profiles of monolayer flakes are taken across the black lines drawn on optical images, and red lines on AFM images. Measured thicknesses correspond well with interlayer distances in dichalcogenide crystals. Observed optical contrast is in the 25-30\% range for $\mathrm{MoS}_{2}$ and $\mathrm{WSe}_{2}$ and is slightly lower that the values predicted in the model. In the case of $\mathrm{NbSe}_{2}$ optical contrast is in the $5-10 \%$ range.

attach a piece of scotch tape to the surface of a bulk crystal. The tape is peeled off together with microscopic fragments of the desired material. It is then rubbed across an $\mathrm{SiO}_{2}$ surface, resulting in mechanical exfoliation of nanolayers that are readily identified in the debris using an optical microscope. In this study we used naturally occurring $\mathrm{MoS}_{2}$ (SPI Supplies) as well as high-quality $\mathrm{WSe}_{2}$ and $\mathrm{NbSe}_{2}$ crystals grown inhouse using the vapor transport method.

After mechanical exfoliation, we image the surface of the sample using an optical microscope (Olympus BX51M) equipped with a color camera (AVT Pike F-505C). After having located the nanolayers with lowest contrast values using the optical microscope, we image the sample using an atomic force microscope (Asylum Research Cypher) in order to measure the nanolayer height using $\mathrm{AC}$ mode imaging. Representative optical and AFM images are shown in figure 4. Based on AFM imaging, we measure the following thicknesses: $6.75 \AA$ for $\mathrm{MoS}_{2}, 6.7 \AA$ for $\mathrm{WSe}_{2}$ and $6.81 \AA$ for $\mathrm{NbSe}_{2}$. These values correspond well to interlayer separation in dichalcogenide crystals, proving that we have managed to exfoliate single layers. Corresponding profiles of optical contrast reported in figure 4 show contrast values for a single layer of $\mathrm{MoS}_{2}$ and $\mathrm{WSe}_{2}$ in the 25-30\% range in the green channel.

By using AFM imaging for the identification of monolayers of dichalcogenides, we were able to make a correspondence with their optical contrast. These experimental values are reported in figure 3. In the case of $\mathrm{WSe}_{2}$ and for $\mathrm{SiO}_{2}$ thicknesses of 90,250 and $270 \mathrm{~nm}$, the contrast is in the $25-30 \%$ range while for 130 and $150 \mathrm{~nm} \mathrm{SiO}_{2}$ thickness, the contrast is negative and is $\sim-10 \%$. Our values show excellent agreement with calculations. In the case of 


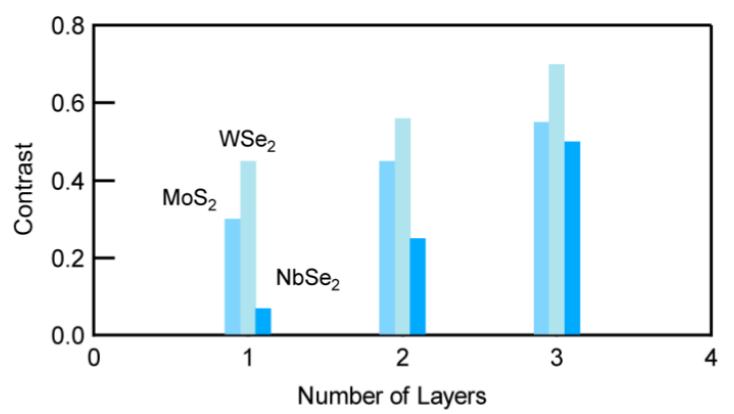

Figure 5. Contrast measured for $\mathrm{MoS}_{2}, \mathrm{WSe}_{2}$ and $\mathrm{NbSe}_{2}$ flakes deposited on $270 \mathrm{~nm} \mathrm{SiO}{ }_{2}$ and containing different numbers of layers identified using AFM. For all three materials, the contrast increases with increasing layer number, indicating that optical imaging can be used to distinguish flakes with differing numbers of layers.

$\mathrm{MoS}_{2}$, the measured contrast values are in good agreement with calculations for $\mathrm{SiO}_{2}$ thicknesses of 90, 130, 150 and $250 \mathrm{~nm}$. However, the measured contrast value at $270 \mathrm{~nm}$ shows a significant discrepancy with respect to the model. Hoping to improve the accuracy of our model we attempted to refine it by considering a very thin layer of water adsorbed between the nanolayer and the substrate. This assumption, however, did not lead to more accurate results. In fact, as water has a very small extinction coefficient, no additional absorption of light takes place and the addition of a water layer only adds a phase factor proportional to its thickness. The observed discrepancy between calculated and observed values of contrast might be due to a variation of optical properties of $\mathrm{MoS}_{2}$ with layer number, such as the recently reported crossover from an indirect gap semiconductor with a bandgap of $1.2 \mathrm{eV}$ [22] to a direct gap material for single layers of $\mathrm{MoS}_{2}[23,24]$ with a bandgap of $1.8 \mathrm{eV}$ [24], making it interesting for the fabrication of transistors with high room temperature current on/off ratios [9].

In the case of $\mathrm{NbSe}_{2}$, the optical contrast of a monolayer is at the limit of visibility and ranges between 5\% and $10 \%$. These values show high discrepancy with respect to the model. This value decreases with time and the monolayer eventually becomes invisible. This may be due to the adsorption of water or oxidation in air [25].

We have also used the AFM to ascertain the thicknesses of 'darker' flakes presumably containing multiple layers. We find that the observed contrast increases with the number of layers, as shown in figure 5. The difference in contrast between double-and triple-layer structures is sufficient to distinguish between them using optical imaging only.

To summarize, we have calculated the expected contrast between thin layers of $\mathrm{MoS}_{2}, \mathrm{WSe}_{2}$ and $\mathrm{NbSe}_{2}$ dichalcogenide crystals and the underlying $\mathrm{SiO}_{2}$ substrate. Contrast in the band corresponding to green light $(495-530 \mathrm{~nm})$ is maximized for $\mathrm{MoS}_{2}$ and $\mathrm{WSe}_{2}$ using 90 and $270 \mathrm{~nm}$ oxide layer thicknesses. High discrepancy with respect to the model is reported for $\mathrm{NbSe}_{2}$. Mechanical exfoliation followed by optical and AFM imaging has confirmed that single and multilayer dichalcogenide nanostructures can be visualized on substrates with proposed oxide thicknesses with easy differentiation between structures containing single, double and triple layers. Optical imaging can therefore be used as a rapid, non-invasive and low cost method for the detection of dichalcogenide nanolayers, paving the way for further studies of these nanomaterials.

\section{Acknowledgments}

Substrate preparation was carried out in the EPFL Center for Micro/Nanotechnology (CMI). This work was financially supported by ERC grant no. 240076 and Swiss SNF grant no. 200021_122044. Single crystals of $\mathrm{WSe}_{2}$ and $\mathrm{NbSe}_{2}$ were grown at the EPFL Crystal Growth facility, financially supported by NCCR MANEP.

\section{References}

[1] Novoselov K S 2004 Science 306666

[2] Frindt R F 1966 J. Appl. Phys. 371928

[3] Novoselov K S et al 2005 Proc. Natl Acad Sci. 10210451

[4] Tenne R and Wold A 1985 Appl. Phys. Lett. 47707

[5] Feldman Y et al 1995 Science 267222

[6] Remskar M et al 2001 Science 292479

[7] Podzorov V et al 2004 Appl. Phys. Lett. 843301

[8] Ayari A et al 2007 J. Appl. Phys. 101014507

[9] Radisavljevic B et al 2011 Nat. Nanotechnol. doi:10.1038/nnano.2010.279

[10] Frindt R F 1972 Phys. Rev. Lett. 28299

[11] Staley N E et al 2009 Phys. Rev. B 80184505

[12] Childres I et al 2010 arXiv:1008.4561 [cond-mat]

[13] Blake P et al 2007 Appl. Phys. Lett. 91063124

[14] Jung I et al 2007 Nano Lett. 73569

[15] Roddaro S et al 2007 Nano Lett. 72707

[16] Ni Z H et al 2007 Nano Lett. 72758

[17] Beal A R and Hughes H P 1979 J. Phys. C: Solid State Phys. 12881

[18] Beal A R, Liang W Y and Hughes H P 1976 J. Phys. C: Solid State Phys. 892449

[19] Beal A R, Hughes H P and Liang W Y 1975 J. Phys. C: Solid State Phys. 84236

[20] Palik E D 1998 Handbook of Optical Constants of solids

[21] Anders H 1967 Thin Films in Optics (London: Focal)

[22] Kam K K and Parkinson B A 1982 J. Phys. Chem. 86463

[23] Splendiani A et al 2010 Nano Lett. 101271

[24] Mak K F et al 2010 Phys. Rev. Lett. 105136805

[25] Vacquier G, Casalot A and Rolland A 1987 Mater. Sci. Eng. 85 L9 